\title{
Effect of Comprehensive Nursing Intervention on the Improvement of Clinical Symptoms and Nursing Satisfaction of Children with Febrile Convulsion
}

\author{
GUO WEI CHEN ${ }^{*}$, FANG XU AND BAO LING ZHOU
}

Department of Pediatrics, Zhongda Hospital, Southeast University, No. 87, Dingjiaqiao, Hunan Road, Nanjing, Jiangsu 210009, China.

Chen et al.: Effect of Comprehensive Nursing Intervention on Children with Febrile Convulsion

To explore the effect of comprehensive nursing intervention on the improvement of clinical symptoms and nursing satisfaction in children with febrile convulsion. 117 children with simple febrile convulsion admitted to our hospital from March 2019 to May 2020 were randomly divided into observation group (59 cases) and control group (58 cases). The control group was given routine nursing intervention, while the observation group was given comprehensive nursing plan. The recovery process, parent's satisfaction with nursing, vital signs, inflammatory factors and neuroendocrine factors before and after nursing were analyzed. The results showed that the seizure stop time, fever abatement time and hospitalization time of the observation group were shorter than those of the control group $(p<0.05)$ and the satisfaction rate of parents was higher than that of the control group $(p<0.05)$; after $2 \mathrm{~d}$ of intervention, heart rate and respiratory rate of the two groups were lower than those before intervention $(p<0.05)$ and those of the observation group were lower than those of the control group $(\mathbf{p}<\mathbf{0 . 0 5})$; in addition, the inflammatory factors Interleukin-6, $\mathrm{C}$-reactive protein, neuroendocrine factors neuron-specific enolase and brain-derived neurotrophic factor of the two groups were lower than those of the control group $(\mathbf{p}<0.05)$. Compared with the control group, although the level of inflammatory factors was significantly lower than that of the control group $(p<0.05)$, there was no significant difference in the level of neuroendocrine factors between the two groups $(p>0.05)$. The application of comprehensive nursing in children with febrile convulsion can improve the quality of nursing and parent's satisfaction, shorten the recovery process of children, more effectively control the level of neuroendocrine factors and inflammatory reaction and promote the recovery of children's vital signs.

Key words: Febrile convulsion in children, comprehensive nursing, nursing satisfaction, inflammatory factors.

Febrile convulsion (FS), as one of the common critical symptoms of the central nervous system in children, mostly occurs in children aged 6 mo to $3 \mathrm{y}$. It is a common pediatric emergency, with a clinical incidence of $4 \% \sim 6 \%$ and the incidence of boys is higher than girls ${ }^{[1]}$. The disease is common in the $12 \mathrm{~h}$ after the appearance of high fever symptoms, which can be manifested as eyeball upturning, teeth closed, mouth foaming, loss of consciousness and other symptoms. In severe cases, it is accompanied by epilepsy symptoms and even death outcomes ${ }^{[2,3]}$. At present, the main clinical treatment for the disease is to control convulsions and body temperature. Therefore, clinical nursing should also start from these two aspects, so as to improve the condition of children. The so-called comprehensive nursing is based on the specific theory, in the process of treatment, according to the high fever of children, combined with effective treatment measures for intervention. Compared with conventional nursing intervention, it is more systematic and specific, can better adapt to clinical needs and improve the quality of nursing service ${ }^{[4,5]}$. Through comprehensive nursing intervention, this study analyzed the nursing effect on children with febrile convulsion. A total of 117 children

*Address for correspondence

E-mail: chenguowei821211@163.com 
with FS treated in hospital from March 2019 to May 2020 were selected as the research objects and randomly divided into observation group (59 cases) and control group ( 58 cases). There were 30 boys and 29 girls in the observation group, with an average age of $(2.2 \pm 0.4) \mathrm{y}$ (range, 6 mo to $5 \mathrm{y}$ ), including 31 cases of bronchitis and 28 cases of rheumatic fever. In the control group, there were 29 boys and 29 girls with an average age of $(2.3 \pm 0.3)$ y (range, 5 mo to 6 y). There were 30 cases of bronchitis and 28 cases of rheumatic fever. Inclusive criteria: all patients met the diagnostic criteria of FS in children; all patients had FS symptoms at the time of treatment; they were 1-3 y old; their guardians informed consent; they were approved by the hospital ethics committee. Exclusion criteria: epilepsy, intracranial infection, organic disease, metabolic disease, genetic disease, poisoning, neonatal convulsion; severe organ dysfunction; other brain diseases. There was no significant difference in general information between the two groups $(\mathrm{p}>0.05)$. The control group was given routine nursing intervention: the children with FS were given first-aid measures for convulsion and physical cooling nursing, and the parents were given FS health education. The observation group added comprehensive nursing on this basis: set up a comprehensive nursing group, which was composed of a clinician, a psychologist and a head nurse as the instructor. The team members were trained, including the concept, content, implementation method of comprehensive nursing, FS first aid and daily nursing points; after the training, the team members were required to find the new progress of FS nursing and treatment at home and abroad, so as to improve the FS first aid nursing and daily nursing process. Finally, the psychiatrists communicate with each other to relieve the negative emotions of parents, observe and pacify the children's emotions. FS emergency care. The children were placed in the lateral position, unbuttoned or stripped of the collar, and pulled up the bed rails on both sides to avoid accidental falling from the bed; the children with serious illness were restrained by the restraint belt; the oral and nasal secretions of the children were removed, and the oxygen concentration was $1.0 \sim 1.5 \mathrm{l} / \mathrm{min}$ through bilateral nasal catheters; The mask should not cover the children's eyes during oxygen inhalation. If the children have asphyxia symptoms, the secretions in the children's mouth and nose should be removed quickly, and the mask should be connected with the air bag for positive pressure ventilation and the effect should be observed. Observe whether the child has tongue falling back. If so, place oropharyngeal ventilation tube; use appropriate venous catheter, quickly establish venous passage through trocar, observe whether the puncture site is red and swollen, and whether the trocar is firmly fixed, so as to avoid the trocar shifting during the onset of the disease. According to the doctor's advice, the children should be treated with anticonvulsant and antipyretic drugs such as diazepam and ibuprofen through vein and the injection speed should be slow when injecting diazepam, so as to avoid short-term repeated medication and increase the risk of side effects. In addition, on the basis of drug treatment, physical cooling therapy should be combined in time, such as placing ice bags or cooling stickers. When using ice bags to cool down, close attention should be paid to the temperature of the children and the skin where the ice bags touch, so as to avoid frostbite and ice compress should be stopped when the temperature drops to $38^{\circ}$. If $30 \%$ alcohol is used to wipe the body of the child, parents should be asked if the child has a history of alcohol allergy before alcohol wiping. Wipe the armpit, elbow socket and groin of the child and pay attention to avoid the chest, abdomen, back neck and sole of the foot. In the process of wiping, observe the vital signs, consciousness, breathing, etc. in case of abnormal breathing and pale complexion, stop wiping immediately and take measures. Diet nursing: children with high calorie, high protein and high cellulose diet, avoid eating raw, cold, greasy food. Children with dehydration caused by high fever can appropriately increase their liquid and semi liquid diet and ask their parents to supplement more water and electrolytes for them. Children who do not like drinking water can also drink fresh vegetable juice. Children's psychological nursing: young children should take interest induction method, play music, animation, animal video and other ways to attract their attention, reduce discomfort. For the older children with certain understanding ability, we should pay attention to listen to them and keep a gentle tone to gain the trust of the children. In the process of nursing, the action should be gentle, use simple and easy to understand language to explain the nursing purpose for the children and encourage the children to use positive language in order to obtain their cooperation. When asking questions, try to use open-ended questions to master the rhythm of communication; guide parents to comfort and encourage children, such as touching, hugging, playing with toys, etc., and parent's company during hospitalization can improve children's sense of security. Health education 
and psychological intervention for parents. The six step standard communication process combined with written materials (disease knowledge manual, etc.) is applied to educate parents about FS etiology, risk factors and preventive measures. Preventive measures are the key points of education. When explaining this part, we should describe in detail what we are going to do, why we are doing this, what impact it has on children, etc., and patiently listen to parent's expression and understand parent's feelings in the process of communication; Ask parents about their doubts and concerns and answer patiently. In this process, parents should try to eliminate their misconception of FS to avoid harm to children. The recovery process (seizure stop time, antipyretic time, hospital stay) of the two groups were compared. Seizure stop time refers to the disappearance of FS symptoms in children and no related symptoms appear again within $72 \mathrm{~h}^{[6]}$. Parent's satisfaction with nursing care. Parents satisfaction was evaluated by their guardians subjective impression and divided into four grades: dissatisfied, general, satisfied and very satisfied. Satisfaction rate $=($ satisfied + very satisfied)/total cases $\times 100 \%$. Vital signs (heart rate, respiration rate) were evaluated before and $2 \mathrm{~d}$ after intervention. The levels of interleukin-6 (IL-6) and C-reactive protein (CRP) were detected by double antibody sandwich enzyme-linked immunosorbent assay (ELISA) before and after treatment. In the morning, $10 \mathrm{ml}$ of venous blood was drawn from fasting state and the serum was separated by centrifugation
(3500 R/min, $10 \mathrm{~min}$ ) to detect the above indicators. The levels of neuroendocrine factors (NSE, BDNF) were measured. The levels of neuron specific enolase (NSE) and brain-derived neurotrophic factor (BDNF) were measured by enzyme-linked immunosorbent assay (ELISA). All data were analyzed by SPSS 20.0. T test was used to compare the measurement data of normal distribution. Rank sum test was used to compare the measurement data of non-normal distribution. The count data were compared by chi square test. $p<0.05$ was considered statistically significant The seizure stopping time, fever abating time and hospitalization time of the observation group were significantly shorter than those of the control group $(\mathrm{p}<0.05)$, as shown in Table 1. Two days after the intervention, HR and RR of the two groups were lower than before the intervention $(p<0.05)$ and the observation group was significantly lower than the control group $(\mathrm{p}<0.05)$, as shown in Table 2. The results showed that the satisfaction rate of parents in the observation group was significantly higher than that in the control group $(\mathrm{p}<0.05)$, as shown in Table 3. Two days after intervention, IL6 and CRP in the two groups were lower than those before intervention $(p<0.05)$ and those in the observation group were significantly lower than those in the control group $(\mathrm{p}<0.05)$, as shown in Table 4 . After $2 \mathrm{~d}$ of intervention, the levels of NSE and BDNF in the two groups were lower than those before intervention $(\mathrm{p}<0.05)$. However, there was no significant difference between the two groups ( $\mathrm{p}>0.05$ ), as shown in Table 5 .

TABLE 1: COMPARISON OF RECOVERY PROCESS BETWEEN THE TWO GROUPS

\begin{tabular}{lcccc}
\hline Group & $\mathrm{N}$ & Seizure stop time $(\mathrm{min})$ & Antipyretic time $(\mathrm{h})$ & Hospital stay $(\mathrm{d})$ \\
\hline Observation group & 59 & $0.54 \pm 0.07$ & $24.58 \pm 2.69$ & $6.78 \pm 1.33$ \\
Control group & 58 & $0.81 \pm 0.12$ & $38.74 \pm 3.05$ & $9.80 \pm 1.74$ \\
$\mathrm{t}$ & 11.663 & 13.472 & 7.239 \\
$\mathrm{p}$ & $<0.001$ & $<0.001$ & $<0.001$ \\
\hline
\end{tabular}

TABLE 2: COMPARISON OF VITAL SIGNS BETWEEN THE TWO GROUPS BEFORE AND AFTER INTERVENTION

\begin{tabular}{lccccc}
\hline \multirow{2}{*}{ Group } & N & \multicolumn{2}{c}{ HR } & \multicolumn{2}{c}{ RR } \\
\cline { 2 - 6 } & & Before treatment & After treatment & Before treatment & After treatment \\
\hline Observation group & 59 & $125.22 \pm 14.57$ & $111.08 \pm 4.93^{*} \#$ & $35.38 \pm 4.53$ & $27.40 \pm 3.05^{*} \#$ \\
Control group & 58 & $124.09 \pm 13.75$ & $116.02 \pm 5.11^{*}$ & $35.10 \pm 4.44$ & $29.85 \pm 3.73^{*}$ \\
\hline
\end{tabular}

Note: compared with before intervention, * was $p<0.05$; compared with the control group, \# was $p<0.05$. 
TABLE 3: COMPARISON OF PARENTS' SATISFACTION WITH NURSING

\begin{tabular}{|c|c|c|c|c|c|}
\hline Group & $\mathbf{N}$ & very satisfied & satisfied & dissatisfied & Satisfaction rate \\
\hline Observation group & 59 & 31 & 27 & 1 & $98.31 \%$ \\
\hline Control group & 58 & 24 & 23 & 9 & $84.21 \%$ \\
\hline $\mathrm{t}$ & & & & & 18.83 \\
\hline $\mathrm{p}$ & & & & & $<0.001$ \\
\hline
\end{tabular}

TABLE 4: COMPARISON OF INFLAMMATORY FACTORS BETWEEN THE TWO GROUPS BEFORE AND AFTER INTERVENTION

\begin{tabular}{lccccc}
\hline \multirow{2}{*}{ Group } & \multirow{2}{*}{ N } & \multicolumn{2}{c}{ IL6 $(\mathrm{ng} / \mathrm{L})$} & \multicolumn{2}{c}{ CRP $(\mathrm{mg} / \mathrm{L})$} \\
\cline { 3 - 6 } & & Before treatment & After treatment & Before treatment & After treatment \\
\hline Observation group & 59 & $10.54 \pm 2.28$ & $5.23 \pm 1.44^{*} \#$ & $7.81 \pm 2.16$ & $4.28 \pm 1.04^{*} \#$ \\
Control group & 58 & $10.43 \pm 2.44$ & $7.87 \pm 1.53^{*}$ & $7.85 \pm 1.94$ & $6.16 \pm 1.56^{*}$ \\
\hline
\end{tabular}

Note: compared with before intervention, * was $p<0.05$; compared with the control group, \# was $p<0.05$.

TABLE 5: COMPARISON OF NEUROENDOCRINE FACTORS BETWEEN THE TWO GROUPS BEFORE AND AFTER INTERVENTION

\begin{tabular}{lccccc}
\hline \multirow{2}{*}{ Group } & \multirow{2}{*}{ N } & \multicolumn{2}{c}{ NSE $(\mathrm{ng} / \mathrm{L})$} & \multicolumn{2}{c}{ BDNF $(\mu \mathrm{g} / \mathrm{L})$} \\
\cline { 3 - 6 } & & Before treatment & After treatment & Before treatment & After treatment \\
\hline Observation group & 59 & $23.11 \pm 5.25$ & $11.16 \pm 3.23^{*}$ & $19.44 \pm 5.17$ & $9.33 \pm 2.50^{*}$ \\
Control group & 58 & $24.08 \pm 5.68$ & $12.24 \pm 3.01^{*}$ & $19.87 \pm 5.11$ & $10.62 \pm 2.96^{*}$ \\
\hline
\end{tabular}

Note: compared with before intervention, ${ }^{*}$ was $p<0.05$.

FS in children are mainly caused by respiratory tract infection, accounting for about $30 \%$ of convulsions in children ${ }^{[7]}$, mainly due to the incomplete development of the central nervous system in children and abnormal discharge of brain nerves after the temperature rises to $38^{\circ[8]}$. In addition to drug treatment, it is very important to take effective nursing measures to improve the condition of children. Comprehensive nursing intervention is a widely used and effective nursing method, which is more systematic and comprehensive than conventional nursing intervention ${ }^{[9]}$. In this study, a comprehensive nursing team was set up before the intervention, and the team members were trained, which is helpful to improve the nurses' cognition of comprehensive nursing and their attitude towards comprehensive nursing. In addition, we included clinicians and head nurses as instructors in the group, which indicated that the supportive attitude towards comprehensive nursing was conducive to creating a good atmosphere for nurses to implement comprehensive nursing. The results showed that the stopping time of convulsion, the time of fever abatement and the length of hospital stay of the observation group were shorter than those of the control group ( $\mathrm{p}<0.05$ ), indicating that comprehensive nursing for FS children is conducive to shorten the recovery time of children. According to the characteristics of
FS as an emergency disease, FS nursing is divided into two parts: emergency nursing and daily nursing, which is helpful for nurses to clarify the priority of nursing intervention measures, improve the process of FS emergency nursing and make emergency treatment more efficient. In this study, the team members were asked to extensively consult the literature related to febrile children and FS, so as to determine the respiratory care and antipyretic intervention measures for children with FS and formulate the intervention plan. Mask oxygen supply and nasal catheter oxygen supply are two commonly used noninvasive oxygen supply methods in clinic, but nasal catheter oxygen supply fits the mask around the mouth and nose and is fixed on the head and face with bandage, which has a sense of restraint and occlusion and the compliance of children is poor ${ }^{[10]}$. In order to reduce the discomfort of children with FS, bilateral nasal catheters were preferred in this study. Physical cooling is a common antipyretic technique for patients with high fever, including warm water bath, alcohol bath and ice compress. Ice compress is an important measure to reduce brain temperature and protect brain cells in children ${ }^{[11]}$. However, alcohol volatilizes quickly, which can play a role of rapid cooling. Therefore, ice compress and alcohol bath were selected as physical antipyretic methods in 
this study. After $2 \mathrm{~d}$ of intervention, $\mathrm{HR}$ and $\mathrm{RR}$ in the observation group were significantly lower than those in the control group $(\mathrm{p}<0.05)$. NSE and BDNF are important indicators to evaluate brain injury ${ }^{[12]}$. The results of this study show that the levels of NSE and BDNF related to brain injury in the observation group are more obvious after intervention, which is related to higher quality of comprehensive nursing and shorter duration of symptoms in FS children. In addition, interleukin-6 (IL-6) is a pleiotropic cytokine with a wide range of functions. IL-6 can regulate the growth and differentiation of a variety of cells, regulate immune response, acute phase response and hematopoietic function and play an important role in the body's anti infection immune response ${ }^{[13]}$. CRP is an acute phase protein synthesized by the liver and its serum level can reflect the degree of inflammatory reaction $^{[14]}$. The results show that the levels of serum CRP and IL-6 in the experimental group after treatment were significantly lower than those in the control group, suggesting that comprehensive nursing can help to reduce the inflammatory reaction, improve the clinical symptoms of children and promote the recovery process of children. In conclusion, the application of comprehensive nursing in children with FS is conducive to improve the quality of care and parents' satisfaction, shorten the recovery process of children, more effectively control the level of neuroendocrine factors and inflammatory reaction and promote the recovery of children's vital signs.

\section{Acknowledgement:}

This work was supported by the Zhongda Hospital, Southeast University.

\section{Conflict of interests:}

The authors report no conflicts of interest.

\section{REFERENCES}

1. S F Al-Ajlouni, I H Kodah. Febrile convulsions in children. Saudi Med J 2000;21(7):617-21.

2. Calderón-González R, Vallejo-Moreno D, Carrera-Sandoval JP, Sevilla-Castillo R, de la Peña-Saucedo F. Febrile convulsion; A clinical study of 303 patients. Bol Med Hosp Infant Mex 1990;47(1):7-13.

3. Caplan E, Dey I, Scammell A, Burnage K, Paul SP. Recognition and management of seizures in children in emergency departments. Emerg Nurse 2016;24(5):30-8.

4. Dunlop S, Taitz J. Retrospective review of the management of simple febrile convulsions at a tertiary paediatric institution. J Paediatr Child Health 2005;41(12):647-51.

5. Hampers LC, Spina LA. Evaluation and management of pediatric febrile seizures in the emergency department. Emerg Med Clin 2011;29(1):83-93.

6. Martin-Fernandez JJ, Molto-Jorda JM, Villaverde R, Salmeron P, Prieto-Munoz I, Fernández-Barreiro A. Risk factors in recurrent febrile seizures. Neurol J 1996;24(136):1520-4.

7. Mustafić N, Tahirović H, Trnovčević J. Frequency of recurrent convulsions after a first febrile seizure: two-year observation results. Med J 2010;132(7-8):227-31.

8. Mustafić N, Tahirović H, Trnovcević J, Kapidzić A. Clinical characteristics at onset of first febrile convulsions. Acta medica Croatica: J Croatian Academy Med Sci 2008;62(5):511-5.

9. Offringa M, Derksen-Lubsen G, Bossuyt PM, Lubsen J. Risk factors for the occurrence of recurrent convulsions following an initial febrile convulsion. Dutch J Med 1992;136(11):516-21.

10. Paul S P, Kirkham E N, Shirt B. Recognition and management of febrile convulsion in children. Nursing standard (Royal College of Nursing (Great Britain): 1987) 2015;29(52): 36-43.

11. Paul SP, Rogers E, Wilkinson R, Paul B. Management of febrile convulsion in children. Emerg Nurse 2015;23(2):2232.

12. Pearce JL, Mackintosh HT. Prospective study of convulsions in childhood. New Zealand Med J 1979;89(627):1-3.

13. Plöchl E, Laubichler W. Retrospective study of 160 children with febrile convulsions. Klin Padiatr 1992;204(1):16-20.

14. Tanabe T, Hara K, Kashiwagi M, Tamai H. Classification of benign infantile afebrile seizures. Epilepsy Res 2006;70:185-9.

This is an open access article distributed under the terms of the Creative Commons Attribution-NonCommercial-ShareAlike 3.0 License, which allows others to remix, tweak, and build upon the work non-commercially, as long as the author is credited and the new creations are licensed under the identical terms

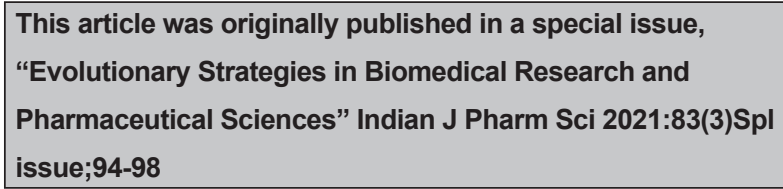

